\title{
Abdominal pain
}

RJ Morgan, D O'Leary, JM Haworth, AZ Demian

\section{The patient}

A 65-year-old man presented with a three-day history of dull epigastric pain and vomiting, followed by persistent retching. Endoscopy demonstrated oesophagitis with linear ulceration, and a sliding hiatus hernia beyond which it was impossible to pass. The patient's symptoms were not improved by ranitidine. His pain worsened, and his abdomen became distended and tender.

Southmead Hospital, Westbury-on-Trym, Bristol BS10 5NB, UK

Department of

Surgery

RJ Morgan

D O'Leary

AZ Demian

Department of

Radiology

JM Haworth

\section{Correspondence to}

Mr RJ Morgan, University

Department of Surgery,

Bristol Royal Infirmary,

Bristol BS2 8HW, UK

Accepted 15 February 1995

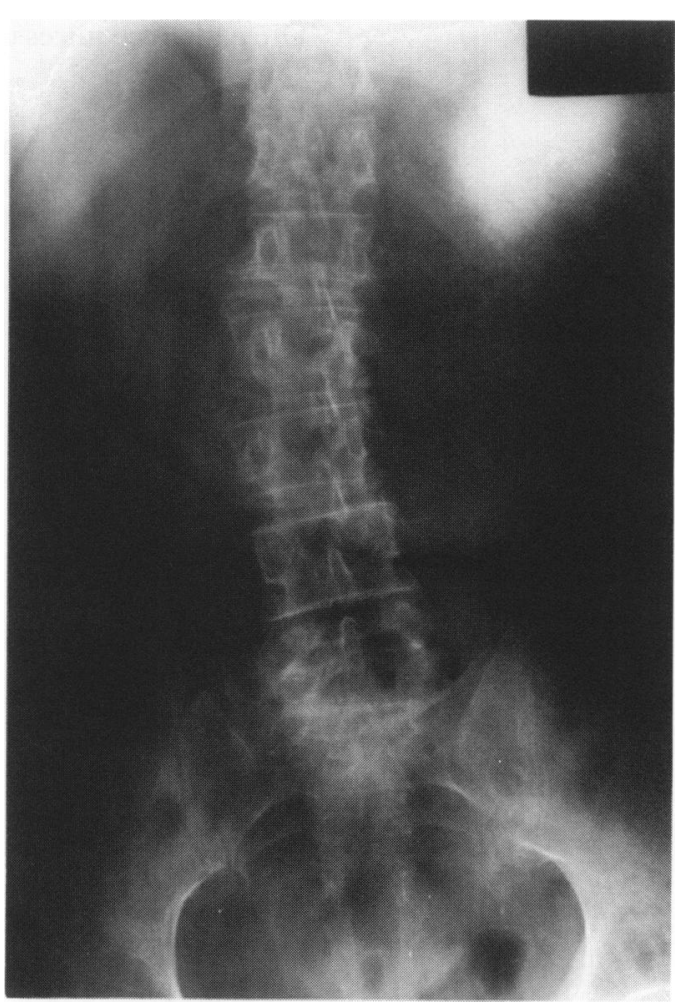

Figure 


\section{Answers}

\section{QUESTION 1}

The stomach is grossly distended. In addition, there is air within the stomach wall. The clinical picture and distribution of air suggest raised intragastric pressure and gastric emphysema. The differential diagnosis is emphysematous gastritis.

QUESTION 2

The patient requires urgent gastric decompression.

In this case a barium study suggested distal gastric obstruction within a para-oesophageal hiatus hernia. Laparotomy revealed volvulus of the gastric antrum within a para-oesophageal hernia with compression of the proximal stomach, causing gastric inlet and outlet obstruction. The hernia was reduced and the defect repaired. The body of the stomach was crepitant, but viable. The patient made an uncomplicated recovery.

\section{Discussion}

Gastric emphysema is a rare radiological finding in which gas is observed in a linear pattern, apparently following tissue planes, within the stomach wall. ${ }^{1}$ It is caused by raised intragastric pressure forcing air into the sub-

1 Lee S, Rutledge JN. Gastric emphysema. Am $\mathcal{f}$ Gastroenterol 1984; 79: 899-904.

2 Badrinath $\mathrm{K}$, Jairam N, Ravi HR, Augustine T Gastric emphysema following drug-induced gastritis. $\mathrm{Br}$. $\mathrm{f}$ Surg 1992; 79: 320. mucosa or subserosal plane. The increased pressure may be due to:

- gastric outlet obstruction

- vomiting

endoscopic insufflation

There may be an associated breach in mucosal integrity, eg, an ulcer or erosion.

Provided that the intragastric pressure is reduced, gastric emphysema usually resolves, often without recourse to surgery. ${ }^{1,2}$ In the present case gastric emphysema probably reflected ischaemia of the stomach wall and increased intragastric pressure consequent on a 'closed loop' obstruction, which was not amenable to non-operative decompression.

Gastric emphysema must be differentiated from emphysematous gastritis. The latter is caused by gas-forming organisms infecting the stomach wall. Patients tend to have evidence of sepsis, and radiographs may show thickening of the stomach wall, with intramural gas distributed in bubbles, streaks and mottled radiolucencies, rather than along tissue planes. ${ }^{1,3}$ Management involves intravenous fluids and nutritional support, with broadspectrum antibiotics. Surgery is required in the event of perforation.

\section{Final diagnosis}

Gastric emphysema caused by an obstructed para-oesophageal hiatus hernia.

Keywords: gastric emphysema, hiatus hernia

3 Moosvi AR, Saravolatz LD, Wong DH, Simms SM. Emphysematous gastritis: case report and review. Rev Infect Dis 1990; 12: 848-55. 